\title{
La police et les « jeunes de cité »
}

\section{Éric Marlière}

\section{Citer ce document / Cite this document :}

Marlière Éric. La police et les « jeunes de cité ». In: Agora débats/jeunesses, 39, 2005. L'animation et ses analogies : des enjeux pour l'action collective. pp. 94-104;

doi : 10.3406/agora.2005.2250

http://www.persee.fr/doc/agora_1268-5666_2005_num_39_1_2250

Document généré le 28/07/2017 


\section{Résumé}

Les rapports entre les jeunes de cité et la police ont toujours suscité un intérêt pour les sociologues. Cet article retrace les principaux travaux effectués sur la question en essayant de les éclairer par un travail empirique réalisé dans une cité HLM de la proche banlieue parisienne. L'enquête ethnographique retracée ici montre que les interactions entre les «jeunes de banlieue» - qu'ils soient diplômés ou marginalisés, salariés ou petits délinquants •avec les représentants des forces de l'ordre sont toujours estampés d'un conflit latent. Le contrôle de police est toujours vécu comme une forme de répression et d'injustice pour ces jeunes qui voient les policiers comme les garants d'un ordre social d'une société profondément injuste.

\section{Abstract}

ple who consider the police officers as the guarantors of the social order of a deeply unjust society.

\section{Resumen}

La policía y los «jóvenes de los bloques»

Las relaciones entre los jóvenes de los bloques y la policía siempre han suscitado un interés para los sociólogos. Este artículo describe los principales trabajos realizados sobre la cuestión intentando aclararlos por un trabajo empírico realizado en un bloque de viviendas de protección oficial del extrarradio parisino cercano. La encuesta etnográfica descrita aquí muestra que las interacciones entre los «jóvenes del extrarradio» - que sean diplomados o marginados, asalariados o pequeños delincuentes $\cdot$ con los representantes de las fuerzas del orden siempre están marcadas por un conflicto latente. Siempre se vive el control de policía como una forma de represión y de injusticia para estos jóvenes que ven a los policías como los garantes de un orden social de una sociedad profundamente injusta.

\section{Zusammenfassung}

Die Polizei und die "Jugendlichen der Siedlungen"

Die Beziehungen zwischen den Jugendlichen der Siedlungen und der Polizei sind für die Soziologen immer von Interesse gewesen. In diesem Artikel werden die wichtigsten Arbeiten, die sich mit diesem Thema befasst haben, erwähnt und dabei wird versucht, sie unter dem Blickwinkel einer empirischen Arbeit, die in einer Siedlung von Sozialwohnungen im nahen Pariser Raum durchgeführt wurde, zu betrachten. Die hier dargestellte ethnographische Untersuchung zeigt, dass die Interaktionen zwischen den •Jugendlichen der Vorstädte" • seien sie mit Diplom oder ausgegrenzt, Lohnempfänger oder kleine Straftäter •und den Vertretern der Polizei immer mit potentiellen Konflikten beladen sind. Die polizeiliche Kontrolle gilt immer als eine Art von Bestrafung und Ungerechtigkeit für diese Jugendlichen, die in den Polizisten die Hüter einer sozialen Ordnung einer extrem ungerechten Gesellschaft sehen.

\section{$\circledast \circledast \Theta$ gamenten}




\section{LA POLICE "JEUNES DE CITE "}

Les rapports entre les jeunes de cité et la police ont toujours suscité un intérêt pour les sociologues. Cet article retrace les principaux travaux effectués sur la question en essayant de les éclairer par un travail empirique réalisé dans une cité HLM de la proche banlieue parisienne. L'enquête ethnographique retracée ici montre que les interactions entre les " jeunes de banlieue " - qu'ils soient diplômés ou marginalisés, salariés ou petits délinquants - avec les représentants des forces de l'ordre sont toujours estampés d'un conflit latent. Le contrôle de police est toujours vécu comme une forme de répression et d'injustice pour ces jeunes qui voient les policiers comme les garants d'un ordre social d'une société profondément injuste.

\section{Éric Marlière}

Docteur en sociologie, chercheur associé au Centre de recherches sociologiques sur le droit et les institutions pénales (CNRS-UMR 2190 et ministère de la Justice) et chargé de cours à l'université Paris-XIII CESDIP

Immeuble Edison - 43, boulevard Vauban - 78280 Guyancourt

Courriel : eric-74@tiscali.fr 
Le thème concernant les formes d'interactions entre les jeunes de cité et la police n'a cessé de défrayer la chronique depuis plus de vingt ans. Polarisant un discours entre des "jeunes de cité " identifiés comme insociables, sauvageons, rebelles et prompts à créer du désordre et une police perçue comme raciste, violente, faisant usage d'une autorité exacerbée favorisant interpellations arbitraires et contrôles au faciès. Certes, la police se révèle plus répressive à l'égard des " jeunes de cité » et des étrangers'. Pour aller plus loin, la démographe M. Tribalat n'hésite pas à affirmer que les " jeunes des cités " perçoivent la police comme une institution " raciste " au service de l'État et des "bourgeois" ". Et même si une partie de ces jeunes ont un emploi et ne connaissent pas de difficultés économiques et sociales particulières, leur présence dans la société en tant qu'enfants d'immigrés, "beurs " des cités, jeunes musulmans, est souvent perçue par les autorités policières comme un facteur de désordre ${ }^{3}$. Bien entendu, les tensions sont rarement absentes lors d'interactions ${ }^{4}$ et les protagonistes - jeunes et policiers - utilisent divers stratagèmes pour tenter de " normaliser " les rapports sociaux afin d'éviter tout débordement. Les policiers disposent du " monopole de la violence légitime ", pour citer Max Weber, alors que les jeunes n'ont que très peu de moyens pour faire face au contrôle au faciès ou à différentes formes d'humiliation dont ils sont les premières victimes.

L'objectif de cet article est de montrer que les rapports entre les jeunes d'une cité et les institutions policières sont marqués parfois par de la violence, souvent par de I'opposition que je qualifierai de symbolique ${ }^{5}$ ou encore tout simplement par du mépris. Mais ces formes d'interaction ne sont guère "neutres". En effet, au cours d'une étude ethnographique menée sur plusieurs années, mêlant à la fois méthode historique, entretiens sociologiques classiques et outils empiriques dont l'observation participante, nous avons pu appréhender les pratiques spatiales des jeunes où les interactions avec les forces de l'ordre font partie du quotidien. Précisons d'emblée que la cité étudiée n'est pas un lieu où règnent violence et atmosphère de " désordre ": cette ancienne cité ouvrière bénéficie d'une bonne réputation localement auprès des travailleurs sociaux et même de la police ; ce n'est nullement un territoire "perdu de la République " où les institutions et autres corporations institutionnelles telles que les pompiers, les ambulanciers ou les facteurs seraient constamment menacés ou malmenés par les habitants. De plus, les jeunes de cette cité connaissent des parcours hétérogènes : un tiers d'entre eux a réalisé des études supérieures et un peu moins d'un quart a des activités que l'on pourrait qualifier de délinquantes $^{6}$, ce qui évidemment n'est pas négligeable. Ainsi, la perception

1. Monjardet D., Ce que fait la police : sociologie de la force publique, La Découverte, Paris, 1996.

2. TRiBalat M. , Dreux : voyage au cœur du malaise français, La Découverte, Paris, 1999.

3. Avenel C., Sociologie des " quartiers sensibles ", Armand Colin, Paris, 2004.

4. Renouard J.-M., "Les relations entre la police et les jeunes : la recherche en question ", Déviance et société, vol. $17, \mathrm{n}^{\circ} 4$, pp. 419-438.

5. J'entends par " symbolique " des pratiques sociales ou culturelles dont le sens structure les rapports sociaux, fédère une perception commune, c'est-à-dire qui construisent une idée du monde et des symboles. Lire LANTZ P., L'investissement symbolique, Presses universitaires de France, Paris, 1996.

6. MARLIĖRE É., Jeunes en cité : diversité des trajectoires ou destin commun ?, L'Harmattan, coll. « Débats Jeunesses ", Paris, 2005. Cet article s'appuie plus précisément sur un travail de thèse soutenue en 2003 portant sur les transformations culturelles chez les enfants d'ouvriers et d'immigrés dans un ancien quartier de banlieue rouge. 
extrêmement négative que peuvent avoir la plupart des jeunes concernant la police est intéressante pour appréhender la nature des rapports qui peuvent exister entre ces enfants d'ouvriers et les institutions.

Les séquences qui vont être exposées ici reconstituent des interactions qui ont eu lieu entre les jeunes et moins jeunes (les âges peuvent varier de 18 à 35 ans) et la police à la fin des années 1990. L'observation de ces interactions montre que, quels que soient les jeunes - étudiants, délinquants, salariés -, la nature des relations avec la police ne peut être neutre en raison des tensions et des enjeux. En d'autres termes, les jeunes observés construisent, en raison de la discrimination, de la stigmatisation et de la ségrégation dont ils sont continuellement l'objet (notamment de la part de la police), un regard critique, distancié et surtout cynique sur les pratiques policières mais aussi envers la société française et la République. L'analyse des rapports entre les jeunes et la police nous interpelle sur plusieurs points. En quoi la description d'interactions entre les jeunes d'une cité et la police est-elle éclairante sur les rapports conflictuels que peuvent avoir ces jeunes avec les institutions? Et surtout comment appréhender les propos de ces jeunes lorsqu'ils ont un regard défaitiste et craintif sur la société qui les entoure ?

En six mois d'enquête intensive sur les lieux, j'ai pu remarquer quelques interactions notables entre les jeunes de cette cité et les forces de l'ordre. Nous allons en restituer quatre : la première, et la plus intense, confronte les jeunes qui viennent d'obtenir la majorité civile et les policiers de la BAC (brigade anticriminalité) ; la deuxième met en scène un contrôle de la brigade des stupéfiants et des petits vendeurs de cannabis âgés d'une vingtaine d'années ; la troisième oppose des CRS contrôlant les papiers de jeunes, étudiants et jeunes salariés habitant la cité ; la dernière scène montre un exemple de provocation policière envers de jeunes adultes âgés d'une trentaine d'années. Enfin, la restitution d'entretiens auprès des jeunes nous permettra de dégager une sorte de vision politique des institutions d'une manière générale.

\section{LES POST-ADOLESCENTS ET LA BAC}

Le premier exemple d'interaction, de loin le plus intense au moment de l'enquête, met en scène un conflit entre des post-adolescents (âgés de 17 à 19 ans plus précisément) et les forces de police. Ces jeunes s'occupaient d'un chien vagabond qui errait dans l'enceinte de l'immeuble; ils semblaient s'accommoder de sa présence et lui achetaient à manger sans lui trouver un lieu précis où dormir. Ce chien imposant se déplaçait librement sans collier et sans laisse dans l'espace résidentiel ; sa présence inquiétait les locataires de la cité qui ont probablement appelé la police au bout de quelques jours. Ainsi, un début d'après-midi, trois policiers en civil et cinq en uniforme arrivent dans le hall $n^{\circ} 10$, lieu où se trouvaient précisément ces jeunes et le chien. D'un ton ferme, les policiers demandent aux jeunes de sortir du hall pour neutraliser l'animal. Mais les jeunes ne l'entendent pas de cette oreille et ne coopèrent qu'à moitié : tous ne veulent pas sortir. Voyant la résistance à moitié passive des post-adolescents, un des policiers sort une arme à feu (pour impressionner les jeunes ou endormir l'animal ?), mais un jeune situé sur sa droite le bouscule violemment.

"Attends, ils sont marrants, ils débarquent, ils nous insultent et puis y'en a un, il sort un flingue comme les shérifs devant des enfants dans la cour! Ils se prennent pour qui ?! »(G., 18 ans, lycéen en bac pro.) 
« Je sais pas ce qui leur a pris... C'est des locataires d'ici, des balances qui les ont appelés. Le chien, il aurait fait de mal à personne mais bon... Qu'est-ce que tu veux, les condés ils viennent pour rien, pour emmerder les jeunes! Alors voilà, maintenant, qu'est-ce que tu veux, nous on avait pas à se laisser faire, c'est la moindre des choses de l'empêcher de tirer !" (H., 19 ans, sans activité.)

Après cet incident, les jeunes présents dans le hall se sauvent en direction de la cité voisine. Les policiers présents sur les lieux, perturbés par cet incident, sont obligés malgré tout de s'occuper en priorité du chien. Mais les jours suivants, des policiers en civil, qui étaient sans doute venus pour l'animal, reviennent fréquemment dans l'espace résidentiel, probablement pour chercher et identifier les jeunes protagonistes présents dans le hall lors de l'incident fâcheux. Et ces jeunes, obligés d'abandonner le chien à la police, ne sont pas en reste et répondent aux rondes policières par des provocations allant du regard insistant à l'insulte :

"Laisse-les tourner comme des cow-boys... De toute façon, j'habite au troisième, si je peux les caillasser de chez moi quand ils seront en voiture, franchement, je le ferai histoire de rigoler un peu. Mais après, ils vont tourner tous les jours, c'est ça le problème. " (F., 18 ans, apprenti stagiaire en mécanique.)

Ce jeune témoin n'en fera rien. Les tensions entre les policiers et les post-adolescents resteront vives avant de s'atténuer progressivement : le quotidien local appelle les policiers de la ville à traiter d'autres affaires plus urgentes et surtout plus graves dans le secteur. Et ces jeunes, qui ne sont pas de véritables délinquants, tâcheront d'oublier cet incident pour vaquer à d'autres occupations à michemin entre l'école, les petits boulots et les petits trafics.

\section{LES DÉLINQUANTS ET LA “ CANINE” "}

Dans cette petite cité, il existe pourtant un petit trafic de cannabis. Ce trafic évolue autour de quelques jeunes seulement : ils sont connus par la police spécialisée, mais celle-ci ne manifeste visiblement pas un grand intérêt pour ces petits trafiquants. Néanmoins, au cours de l'une de mes observations participantes avec deux de ces petits trafiquants âgés d'une vingtaine d'années à l'époque, une brigade de police en voiture - dont la tâche concerne les produits stupéfiants - s'arrête à notre hauteur en nous fixant longuement et en roulant doucement. Puis, tout en faisant un bref demi-tour, les trois policiers présents dans la voiture continuent à nous regarder fixement. Sentant le contrôle de police imminent, un des jeunes trafiquants les regarde à leur tour, tout en conseillant à son camarade de se débarrasser de la marchandise devenue trop encombrante :

«T., je crois qu'ils vont venir nous casser les couilles, ces fils de pute! Balance le teuchi, dès que la voiture va tourner pour faire demi-tour, tu le jettes vers la plante là ! Dès qu'ils vont pas nous regarder ! " (R., 24 ans, espace-nord " délinquant ».)

Son compagnon jette de façon discrète le morceau de cannabis sur les mauvaises herbes qui ont poussé au bord du trottoir. La voiture de police "banalisée " s'arrête effectivement devant nous ; les inspecteurs sortent énergiquement de la voiture en nous entourant et lancent :

"Bonjour, contrôle de police!"

7. Appellation pour définir des policiers en uniforme qui ont pour objectif de chercher de la drogue. La plupart du temps, ils sont accompagnés d'un chien. 
La Canine ${ }^{8}$ en nous encadrant prend possession du lieu et s'intéresse plus particulièrement aux deux individus avec qui je discutais jusqu'à présent ${ }^{9}$.

Lorsque le contrôle de police commence, les petits trafiquants ont recours à des stratagèmes comme le mensonge et l'imposture. II est environ 14 h 30 lorsque la fouille s'effectue : deux policiers scrutent chacun un jeune pendant qu'un autre, éloigné à deux mètres, supervise l'ensemble ${ }^{10}$. Les policiers réclament une pièce d'identité à celui qui a demandé à son camarade de balancer le morceau de cannabis quelques instants auparavant. Ce dernier déclare qu'il n'a pas ses papiers sur lui. Alors, I'un des trois policiers lui demande son nom : celuici triche sur son identité. Perplexes, les policiers cherchent dans les alentours un éventuel morceau de cannabis puis reviennent vers le collègue qui questionne le trafiquant ; I'un des policiers, de plus en plus circonspect par rapport à l'attitude du jeune interrogé, fouille à nouveau ce dernier : il lui demande ouvertement s'il n'a pas jeté le produit dans les buissons. Jusqu'alors épargné par l'interrogatoire, I'autre jeune, pour faire diversion, propose aux policiers de le fouiller également ; visiblement agacé par ce type de requête, un des agents de police lui répond sèchement :

"Toi, tu fermes ta gueule, on te fouillera quand on te le dira, t'as compris?"

En réalité, les policiers qui exercent la fouille ont quelque chose à reprocher au jeune qu'ils sont en train d'inspecter et surtout de suspecter : ils cherchent davantage à l'identifier qu'à savoir s'il possède un produit illégal sur lui. Comme l'expliquera plus tard le "délinquant » rigoureusement contrôlé et questionné ici, un des policiers présents lors de cette intervention l'a reconnu après une " affaire " qui a mal tourné quelques jours plus tôt : le jeune inspecté avait eu affaire à celui-ci lors d'un autre contrôle de police.

" Ils étaient venus contrôler des potes à moi. Moi, ils m'ont pas vu, il faisait nuit et j'étais caché derrière les buissons. J'ai pris deux pierres et je leur ai balancé... Je me suis sauvé, j'ai couru, flippant ! J'ai mis trois heures avant de rentrer chez moi, ils arrêtaient pas de tourner!" (R., 23 ans, sans profession.)

Les policiers en question lui avaient couru après mais sans succès. C'est pourquoi, lors de cette observation participante qui met en scène un contrôle de police, ce petit délinquant est pris au dépourvu : de manière astucieuse et conscient à ce moment qu'il ne peut leur échapper, il donne, pour paraphraser Erving Goffman, " une représentation frauduleuse" " de sa personne puisqu'il triche non seulement sur son identité mais aussi en se montrant poli, affable voire coopératif. Ces interactions comparables à des impostures sont fréquentes, notamment lorsque ces jeunes sont pris de court par les forces de police. Pour ces petits délinquants, la seule porte de sortie est alors de faire "bonne figure " face aux policiers : en faisant semblant de coopérer - en se montrant poli et même affable - avec les

8. Ce jour-là, ces policiers n'avaient pas de chien. À mon sens, il s'agissait plutôt d'une patrouille de routine et d'un travail d'identification et de reconnaissance.

9. Ils m'ont demandé d'une manière sèche de m'éloigner comme s'ils savaient d'emblée que j'étais étranger aux activités des deux jeunes.

10. Généralement, les jeunes enlèvent leur veste, les policiers demandent aussi aux jeunes de vider leurs poches et font des fouilles corporelles. Souvent le doute subsiste : un contrôle de ce type dure environ dix à quinze minutes.

11. Goffman E., La mise en scène de la vie quotidienne : la présentation de soi, Minuit, coll. «Le sens commun ", Paris, 1973. 
représentants de la loi. Toutefois, les petits trafiquants ne sont pas les seuls à user de stratagèmes pour tromper les policiers, même si ces derniers n'ont pas d'autres choix afin d'éviter l'arrestation et donc la " garde à vue ".

\section{Quels que soient les jeunes - étudiants, délinquants, salariés -, la nature des relations avec la police ne peut être neutre en raison des tensions et des enjeux.}

\section{LES ÉTUDIANTS DE LA CITÉ ET LES CRS}

Les " jeunes de cité " qui sont insérés dans le monde du travail ou qui font des études supérieures s'insurgent ouvertement contre les contrôles des CRS dans la cité jugés trop routiniers. Ces jeunes n'hésitent pas à jouer également des rôles pour montrer aux forces de l'ordre que les contrôles sont injustes et surtout arbitraires. Je discutais avec trois jeunes dans l'espace résidentiel en fin d'après-midi lorsqu'un car de CRS passe dans la rue adjacente à l'entrée des immeubles. Le car de CRS passe lentement à notre hauteur et les agents à l'intérieur regardent avec insistance le groupe que nous formons à ce moment.

"Putain, ils sont relous là à téma comme ça ! C'est casse-couilles ! " (K., 20 ans, trafiquant de cannabis.)

"Tu ne les calcules pas! Tu n'y fais pas attention!" (D., 27 ans, chef de piste à Air France.)

Le dernier interlocuteur tente de calmer la situation. Mais au bout de dix mètres, la camionnette fait marche arrière : sortent alors quatre CRS munis de matraques qui viennent dans notre direction.

"Vas-y putain, ils vont nous casser les couilles!" (K., 20 ans, trafiquant de cannabis.)

Le chef de brigade déclare d'un ton calme et posé :

"Bonsoir, contrôle d'identité. "

Deux jeunes confessent qu'ils n'ont pas leurs papiers. Le premier d'entre eux se justifie :

"De toute façon, on habite à moins de 50 mètres d'ici ! " (I., 24 ans, étudiant en licence de biologie.)

Les quatre jeunes CRS s'occupent respectivement des trois jeunes et de moimême : ils nous fouillent tout en nous posant des questions concernant nos activités et nos lieux de résidence. Le brigadier-chef supervise l'ensemble des contrôles tout en essayant de détendre l'atmosphère. Le jeune CRS qui s'occupe de moi, relativement tendu, me demande de vider mes poches et, comme je n'avais pas de papiers sur moi, se saisit de ma carte bleue comme moyen d'identification pour le code terminal installé dans toutes les voitures de police. Très suspect à mon égard, semble-t-il, il revient me fouiller. 
Un jeune, chef de piste à Air France, se met à discuter avec le CRS gradé qui supervise l'opération :

"Monsieur, vous trouvez ça normal ? ! On est en bas de chez soi. Vous nous infligez une telle honte ! C'est I'humiliation ! " (D., 27 ans, chef de piste à Air France.)

Le brigadier-chef tente de relativiser les propos de ce jeune en affirmant que ce n'est pas très grave. Mais le jeune salarié continue sa plaidoirie :

"Ouais mais si ma sœur ou mon père passe, j'ai l'air de quoi ? ! En plus, c'est une cité très calme, on travaille tous ici, monsieur ! " (D, 27 ans, chef de piste à Air France.)

Le chef de la brigade ne répond plus, ce qui n'empêche pas le jeune en question de surenchérir sur son sentiment d'injustice :

"Donc pour vous ici, on est tous des voyous, vous nous prenez pour qui ? !" (D., 27 ans, chef de piste à Air France.)

Le brigadier-chef ne bronche toujours pas. Avec un certain agacement, le chef de piste à Air France conclue son monologue pour exprimer son amertume et sa perception de la justice :

"En fait ici, on est encore au Moyen Âge, il y a une justice à deux vitesses. Allez voir ceux qui magouillent, je sais pas moi !" (D., 27 ans, chef de piste à Air France.)

Le quatrième CRS, celui qui avait pris les papiers pour faire l'inventaire des identités des jeunes fouillés, revient de la camionnette et déclare à son supérieur que nous sommes en règle. Après nous avoir rendu les papiers, les CRS s'en vont rapidement et le brigadier, en guise de salut, fait un hochement de la tête en notre direction. Tout le monde retourne vaquer à ses occupations hormis le délinquant, présent dans le groupe, qui s'inquiète de voir les contrôles se multiplier dans l'espace local et donc s'intéresser à son business :

"Putain si ça continue, ils vont niquer mon business! Ils commencent à me prendre la tête ! " (K., 20 ans, trafiquant de cannabis.)

L'ensemble des jeunes présents lors du contrôle de police, hormis le délinquant, exercent un emploi salarié et se trouvent par conséquent étrangers aux mondes de la délinquance et des trafics divers. C'est ce qui permet au jeune chef de piste à Air France d'exprimer son sentiment d'injustice au CRS gradé en lui déclarant ouvertement son point de vue concernant le contrôle d'identité. Et c'est à cette occasion que ce dernier s'autorise, afin de culpabiliser les CRS, une "représentation frauduleuse " en affirmant que sa sœur et son père sont susceptibles de passer par là à tout moment. En effet, ce jeune habite plus précisément la cité voisine et n'a pas de sœur. L'imposture a pour objectif de dénoncer le contrôle de police comme injuste et arbitraire - et ce jeune salarié peut se le permettre puisqu'il n'a, en l'occurrence, rien à se reprocher vis-à-vis de la loi - alors que dans le cas précédent, le stratagème du mensonge consistait à protéger les "délinquants " d'une éventuelle arrestation en raison de leur activité illégale. Ces observations sont d'autant plus éclairantes dans la mesure où s'est créée une sorte de "cercle vicieux " autour d'une suspicion réciproque et de provocations mutuelles dans les interactions quotidiennes entre jeunes de la cité et police ${ }^{12}$. Et

12. Lagrange H., De l'affrontement à l'esquive, violences délinquances et usages de drogues, Syros, Paris, 2001. 
comme le montre l'exemple suivant, les jeunes voire les résidents en quartier dit défavorisé apparaissent comme une population suspecte aux yeux des policiers.

\section{LES PATROUILLES DE LA BAC ET LES TRENTENAIRES}

Les policiers de la BAC sont particulièrement mal perçus par les jeunes. Ces fonctionnaires de police, souvent jeunes, sont réputés agressifs et violents dans les cités HLM. Un dimanche, en début d'après-midi, cinq jeunes adultes de 30 à 35 ans environ, qui sont pour la plupart mariés et ont une situation professionnelle plus ou moins stable, se retrouvent groupés dans la cité comme à l'accoutumée avant d'aller voir jouer l'équipe municipale. Survient alors une 106 blanche « banalisée ", avec à l'intérieur des inspecteurs qui regardent de façon soupçonneuse les jeunes adultes en pleine conversation; I'un des policiers baisse la vitre de la voiture et lance de manière provocante et surtout impromptue :

"Alors, les petits pédés, ça va ? !"

La 106 accélère brutalement en se dirigeant vers le commissariat du centreville. Les propos du policier suscitent alors surprise et étonnement de la part des trentenaires présents ainsi qu'un ensemble de commentaires :

"Qu'est-ce qui se passe, ils dorment mal au commissariat ou alors ils n'ont pas eu d'augmentations ? !" (L., 34 ans, comptable à la Défense.)

"Ah ! J'avais même pas remarqué que c'était des condés ! Je croyais que c'était un type qu'on connaissait. Ils sont pas bien dans leur tête ! " (A., 32 ans, responsable d'une petite société de transport.)

\section{L'activité policière est appréhendée par ces jeunes comme une pratique institutionnelle qui a pour finalité leur surveillance et leur répression.}

Les jeunes adultes observés manifestent, à l'encontre de ce type de comportement, de l'étonnement, de l'incompréhension, mais surtout de l'indifférence. Dans les travaux de certains sociologues, la BAC se caractérise au sein même de la police par des méthodes d'intervention très ambiguës :

"Strictement nocturnes, les territoires des BAC sont un espace de chasse, peuplé non pas de victimes ou de "branleurs" mais de criminels engagés volontairement dans la transgression ${ }^{13}$."

Le terrain de la BAC n'est pas seulement nocturne puisque nous sommes, au moment de la scène, en début d'après-midi. Les contrats locaux de sécurité signés en partenariat entre le préfet et le maire autorisent l'omniprésence des 
forces de l'ordre, quelles que soient les circonstances. Néanmoins, ce type de comportement conforte la perception négative qu'ont ces jeunes de la police, même parmi ceux qui sont "intégrés " dans la société.

\section{UN REgARD SUR LA POLICE OU LA SOCIÉTÉ FRANÇAISE ?}

Les séquences restituées pour les besoins de l'article nous montrent différents acteurs. Mais elles laissent penser que les rancœurs sont tenaces. La police, que ce soit pour l'étudiant diplômé de la cité, le salarié ou encore le " délinquant ", est perçue comme l'ennemi commun.

"Pour moi, certains policiers ont des comportements de voyous, ils ne valent pas mieux que certains jeunes qui traînent en bas. J'ai l'impression qu'ils sont là pour faire de la surveillance et emmerder les jeunes qui cherchent aussi. Je ne dis pas toute la police mais certains, pour moi, restent de véritables dangers publics avec un flingue et une carte de police " (X, 23 ans, étudiant à l'École supérieure des sciences économiques et sociales.)

Pour cet étudiant en voie de réussite scolaire, la police est très mal perçue : elle est un facteur de désordre et de trouble publics.

"Le rôle de la police est clair. Elle sert l'État et les intérêts de la grande bourgeoisie parisienne qui tient l'État. La police s'est toujours opposée aux ouvriers, aux petits, alors tu vois, les Arabes ne sont pas les plus appréciés dans cette affaire. Les bavures policières sur un Moustapha ou un Rachid en disent long sur la démocratie de ce pays. II faut regarder les choses en face ! Jette un coup d'œil sur la Constitution et tu comprendras mieux le rôle de la police garante de l'ordre bourgeois. " (X, 28 ans, étudiant en thèse de droit à Nanterre.)

La police sert la cause des grands. La démocratie, pour ce thésard issu d'une famille en provenance d'Algérie, n'est qu'un subterfuge, l'agissement de la police républicaine en est la confirmation.

\section{Du racisme immigré des années 1970 et 1980 , nous sommes passés à une stigmatisation féroce concernant les “ jeunes de cité " associés à la culture “ arabo-musulmane ".}

"Tu sais, c'est grillé ! Une fois, j'étais au commissariat, y'a cinq, six ans pour une broutille. Je t'assure les mecs, ils prennent un plaisir à te gifler. Et ça n'avait rien à voir avec la répression, mais c'est comme ça ! Eux, ils ont les lois avec eux, et nous, ben, il nous reste que les sentiments. Pour les flics t'es qu'un bougnoule ! » (X, 29 ans, salarié à la SNCF, représentant syndical FO.) 
Pour ce jeune, aujourd'hui titulaire de son emploi et issu de l'immigration maghrébine, les rapports avec la police restent teintés d'amertume et de ressentiment. Le rôle de la police est donc perverti par ce que l'interrogé ressent comme du racisme de la part des agents représentant les forces de l'ordre. Pour le témoin suivant, le constat est encore plus alarmant :

"Vas-y, c'est un truc de fou! Toi, tu vends une barrette de cannabis et tu te fais arrêter, frapper. On te met des menottes, on t'insulte. Eux, les politiciens, ils détournent des briques et des briques et personne les calcule. Les policiers, ils disent rien parce que c'est les politiques qui ont le power! » (X, 22 ans, sans emploi, sans diplôme, petit trafiquant de cannabis.)

Le constat d'injustice est révélateur de la perception que ce jeune a de la société française. Pour lui, la police l'empêche de vivre alors qu'elle couvre les agissements honteux des politiciens, des riches, bref des puissants. Et le mépris que constitue la police pourrait s'élargir à d'autres institutions voire à d'autres professions ${ }^{14}$.

\section{VIOLENCE DES JEUNES OU VIOLENCE INSTITUTIONNELLE ?}

Ces quelques observations présentées ici montrent que les scènes sont d'autant plus variées que les acteurs sont divers : nous n'avons jamais affaire aux mêmes jeunes, ni aux mêmes policiers, et les contextes d'observation ne se ressemblent pas. Tout d'abord, les rapports sociaux entre ces deux groupes, certes souvent opposés dans leurs intérêts, sont plus complexes que la perception qui circule fréquemment dans le discours commun et invitent, par conséquent, les chercheurs à s'y intéresser davantage ${ }^{15}$. Néanmoins, il semble se dégager un dénominateur commun dans la nature des interactions entre ceux que l'on pourrait dénommer " jeunes habitant la cité " et les forces de police : des rapports conflictuels et d'opposition ${ }^{16}$. Les interactions décrites ici en témoignent puisque les rapports entre jeunes et police oscillent entre pugilat et provocation pour les adolescents majeurs, entre mensonge et dissimulation pour ce qui est des délinquants ou même des salariés et étudiants. La police est alors, au regard des mises en scène présentées, appréhendée comme ennemi commun : quels que soient le contexte ou l'enjeu, les rapports entre les jeunes et la police sont ici teintés de méfiance, de distance et de provocation dont les issues sont parfois incertaines ${ }^{17}$. Ensuite, les propos tenus par les jeunes cachent mal les ressentiments envers la police et les institutions d'une manière générale ; l'activité policière est appréhendée par ces jeunes comme une pratique institutionnelle qui a pour finalité leur surveillance et leur répression. Au vu des faits et des discours observés ici, on se pose la question du traitement policier dont sont quotidiennement l'objet les jeunes étudiés ${ }^{18}$. Car, au-delà de la répression policière qui nous intéresse

14. MARLIĖRE É., «Dispositifs de discipline et logiques de résistance : l'intervention sociale vue par les jeunes d'une cité HLM de Gennevilliers ", Le passant ordinaire, n 44, 2003, pp. 33-36.

15. Esterle-Hedibel M., "Jeunes des cités, police et désordres urbains ", in MuCHIELLI L., Robert P., Crime et sécurité, l'état des savoirs, La Découverte/Syros, Paris, 2002, pp. 376-385.

16. Dubet F., Lapeyronnie D., Les quartiers d'exil, Le Seuil, Paris, 1992. Les auteurs n'hésitent pas à montrer que police et jeunes apparaissent comme des "bandes rivales " en perpétuelle friction. 17. Jobard F., Bavures policières, La Découverte, Paris, 2002.

18. LÉvy R., Zauberman R., “ De quoi la République a-t-elle peur ? Police, Blacks et Beurs ", Mouvements, $n^{\circ} 4$, p. 39-46. 
ici, le traitement institutionnel et surtout médiatique de ces jeunes nous interroge plus sérieusement ${ }^{19}$. Face aux dérives actuelles de plus en plus alarmantes à mon sen $^{20}$, la question de l' " intégration républicaine " doit être reposée en d'autres termes. Nos institutions sont-elles en mesure d'accepter des égaux mais différents comme elles avaient réussi à le faire tant bien que mal par le passé21 ? Et ce notamment dans un contexte de durcissement des lois où l'occupation des halls d'immeuble par des jeunes s'achemine vers le délit pénal ${ }^{22}$ ? La police semble le dernier interlocuteur dans ces quartiers, avec ses objectifs qui lui sont spécifiques: maintenir l'ordre, contrôler, surveiller, réprimer. La prise en charge des problèmes sociaux se trouve trop souvent réduite à la répression ${ }^{23}$.

Les propos rapportés ici de ces jeunes habitant la cité HLM, quels que soient le niveau d'étude, le statut social ou encore l'âge, n'en sont pas moins préoccupants car leurs discours laissent difficilement paraître qu'ils vivent et évoluent dans une société démocratique. Du racisme immigré des années 1970 et 1980, nous sommes passés à une stigmatisation féroce concernant les " jeunes de cité " associés à la culture " arabo-musulmane ". Perçus comme hostiles à la société, ces jeunes font l'objet de " campagnes » intellectuelles, politiques et journalistiques les accusant des pires maux ${ }^{24}$. Et cette situation cristallise les incompréhensions et un sentiment de malaise collectif chez ces jeunes. Cette violence indicible mais implacable à leur égard renforce un sentiment de peur, d'insécurité et de crainte même chez les jeunes de cité qui veulent s'en sortir. C'est pourquoi la présence policière dans leur espace territorial reste appréhendée par eux à la fois comme une violation de leur espace mais surtout comme une menace. Cette situation développe chez ces enfants d'ouvriers et d'immigrés pour la plupart un sentiment d'injustice mais également d'hostilité envers une société qui peine à les accepter.

19. Mucchielli L., Violences et insécurité : fantasmes et réalités dans le débat français, La Découverte, Paris, 2002.

20. Lire en ce sens Jobard F., Zimolag M., " Quand les policiers vont au tribunal : étude sur les outrages, rébellions et violences à agents ", Questions pénales, vol. XVIII/2, mars 2005.

21. NOIRIEL G., État, nation et immigration : vers une histoire du pouvoir, Paris, Belin, 2001.

22. Vulbeau A., “Émergence et usages sociaux des "incivilités" ", Réalités familiales, n 73, 2004, pp. 74-85.

23. L'HeUILLET H., Basse politique, haute police : une approche historique et philosophique de la police, Fayard, Paris, 2001.

24. MucchielLi L., Le scandale des “tournantes » : dérives médiatiques, contre-enquête sociologique, La Découverte, Paris, 2005. 